\title{
Saturation Processes in Nonlinear Media Modeled by Four-Level Configuration with Coincident Absorption and Emission Bands
}

\author{
J.S.M. ADDASI* \\ Applied Physics Department, Faculty of Science, Tafila Technical University, Tafila, Jordan \\ Taiba University, Faculty of Applied Science, Applied Physics Department, Medina, Saudi Arabia
}

(Received February 26, 2011)

\begin{abstract}
The four-level model is used to study the nonlinear media with coincident absorption and emission bands in both principal $\left(\mathrm{S}_{0}-\mathrm{S}_{1}\right)$ and excited $\left(\mathrm{T}_{1}-\mathrm{T}_{2}\right)$ channels. The excitation of molecules occurred by two light fields: radiations with intensity $I_{12}$ at frequency $\omega_{0}$ tuned at the centre of absorption band of principal singlet-singlet channel and radiations with intensity $I_{34}$ at frequency $\omega$ tuned at the centre of absorption band of excited triplet-triplet channel. The monotonic dependence of phase response of media in both principal and excited channels and the saturation processes are studied theoretically and numerically for four-level model.
\end{abstract}

PACS: $78.20 . \mathrm{Bh}, 78.20 . \mathrm{Ci}$

\section{Introduction}

Many nonlinear phenomena in laser physics are based on the study of nonlinear properties of matter. Four-wave mixing, dynamic holograms and other nonlinear processes are interested in phase response of medium [1-3]. The control of nonlinear processes occurs in many works such as: control of optical bistability [4], four-wave mixing controlled by driving fields [1], and reflectivity control of the dynamic PC-holograms [5]. The control of nonlinear processes occurs easily by using double-frequency optical excitation [6].

Complex organic compounds, dye solutions, are characterized by singlet $(\mathrm{S})$ and triplet $(\mathrm{T})$ electronic energy levels [7]. The electronic states contain a set of vibrational sublevels with significantly law life time. The transitions between singlet-singlet and triplet-triplet levels for dye solutions corresponds to absorption in visible or near ultraviolet (infrared) regions of spectrum.

Some theoretical and experimental works study the nonlinear changes in a refractive index caused by transitions of molecules to the excited singlet or triplet levels [7-12]. In many cases the dye solutions can be modeled by three-level or four-level configurations, depending on the parameters of the radiations and medium.

Four-level configuration with two light fields, illustrated in Fig. 1, will be used in this work to model the nonlinear medium. The transitions of molecules from ground state $\left(\mathrm{S}_{0}\right)$ occurred by irradiations with intensity $I_{12}$ at frequency $\omega_{0}$. At the same time the transitions of molecules from the first excited singlet level $\left(\mathrm{S}_{1}\right)$ to the first excited triplet level $\left(\mathrm{T}_{1}\right)$ are radiation or

\footnotetext{
* e-mail: Jihad_addasi@yahoo.com
}

Fig. 1. Diagram of four-level model. The solid lines denote the radiation-induced transitions of molecules, and the dashed lines denote spontaneous and radiationless transitions. $S_{0}$ is the ground state; $S_{1}$ is the first excited singlet level, $\mathrm{T}_{1}\left(\mathrm{~T}_{2}\right)$ are the first (second) triplet excited level; $I_{12}, \omega_{0}\left(I_{34}, \omega\right)$ are the intensity, frequency of radiations in principal (excited) channel.

spontaneous (dashed line in Fig. 1), where the radiation-induced transitions between singlet and triplet levels are spin-forbidden. The excitation of molecules in the excited triplet channel $\left(\mathrm{T}_{1}-\mathrm{T}_{2}\right)$ occurs by radiations with intensity $I_{34}$ at frequency $\omega$.

\section{Theory}

The balance equations under a double frequencies excitation of dye solution modeled by four-level configuration can be written as follows:

$$
\begin{aligned}
& N_{1} B_{12}\left(\omega_{0}\right) I_{12}=N_{2}\left(B_{21}\left(\omega_{0}\right) I_{12}+v P_{21}\right)+N_{3} v P_{31}, \\
& N_{1} B_{12}\left(\omega_{0}\right) I_{12}=N_{2}\left[B_{21}\left(\omega_{0}\right) I_{12}+v\left(P_{21}+P_{23}\right)\right] \\
& N_{3} B_{34}(\omega) I_{34}=N_{4}\left[B_{43}(\omega) I_{34}+v P_{43}\right] \\
& N_{1}+N_{2}+N_{3}+N_{4}=N
\end{aligned}
$$

where $N_{i}$ is the population of the $i$-th energy level, $N$ 
is the number of molecules in the unit volume of nonlinear medium, $P_{i j}=A_{i j}+d_{i j}$ is the total probability of spontaneous and radiationless transitions in the $i-j$ channel, $P_{23}=d_{23}$ (there is no spontaneous transitions between excited singlet level $\mathrm{S}_{1}$ and triplet excited level $\left.\mathrm{T}_{1}\right), v=c / n$ is the light velocity in the nonlinear medium. The Einstein coefficients $B_{12}\left(\omega_{0}\right), B_{21}\left(\omega_{0}\right)$ are determined at the frequency of radiations $\omega_{0}$ in principal singlet-singlet $\left(\mathrm{S}_{0}-\mathrm{S}_{1}\right)$ channel. At the same time $B_{34}(\omega), B_{43}(\omega)$ are determined at frequency of radiations $\omega$ in the excited triplet-triplet $\left(\mathrm{T}_{1}-\mathrm{T}_{2}\right)$ channel.

The refractive index can be determined by using balance equations. In this work we will study the nonlinear medium with coincident absorption and emission bands in both singlet-singlet $\left(\mathrm{S}_{0}-\mathrm{S}_{1}\right)$ channel and triplettriplet $\left(\mathrm{T}_{1}-\mathrm{T}_{2}\right)$ channel. In this case the real part of refractive index equals zero in both channels $\left(n_{12}\left(\omega_{0}\right)=\right.$ $\left.0, n_{34}(\omega)=0\right)$, and the extinction coefficients are

$$
\kappa_{12}\left(\omega_{0}\right)=\frac{\kappa_{0}}{1+\alpha I_{12}}
$$

in the principal singlet-singlet channel $\left(\mathrm{S}_{0}-\mathrm{S}_{1}\right)$ and

$$
\kappa_{34}(\omega)=\frac{\kappa_{34}^{0}}{1+\beta I_{34}}
$$

in the excited triplet-triplet $\left(\mathrm{T}_{1}-\mathrm{T}_{2}\right)$ channel, where $\kappa_{0}=h n N B_{12} / 2$ is the initial extinction coefficient, $\kappa_{34}^{0}=\kappa_{0}\left(P_{23} / P_{31}\right) B_{34} I_{12} / v\left(P_{21}+P_{23}\right) /\left(1+J I_{12}\right)$ is the extinction coefficient in the excited channel at intensity $I_{34}=0, J=\left[B_{12}\left(1+P_{23} / P_{31}\right)+B_{21}\right] / v\left(P_{21}+P_{23}\right)$, $\alpha=\left(J+b I_{34}\right) /\left(1+a I_{34}\right), \beta=\left(a+b I_{12}\right) /\left(1+J I_{12}\right)$, $a=B_{43} / v P_{43}$ and $b=a J+\left(P_{23} / P_{31}\right) B_{12} B_{34} / v^{2}\left(P_{21}+\right.$ $\left.P_{23}\right) P_{43}$.

The saturation intensity is defined as the value of radiation intensity for which the absorption is decreasing at half of its initial value. Using this definition and Eqs. (2) and (3) we can find

$$
I_{12}^{\mathrm{sat}}=\frac{1}{\alpha}=\frac{J+b I_{34}}{1+a I_{34}},
$$

for principal singlet-singlet channel $\left(\mathrm{S}_{0}-\mathrm{S}_{1}\right)$ and

$$
I_{34}^{\text {sat }}=\frac{1}{\beta}=\frac{a+b I_{12}}{1+J I_{12}}
$$

for excited triplet-triplet channel $\left(\mathrm{T}_{1}-\mathrm{T}_{2}\right)$.

\section{Results and analysis}

From Eqs. (4), (5) the saturation intensity in principal singlet-singlet channel $\left(\mathrm{S}_{0}-\mathrm{S}_{1}\right)\left(I_{12}^{\text {sat }}\right)$ decreases with the increase of radiation intensity $I_{34}$ in the excited triplettriplet channel $\left(\mathrm{T}_{1}-\mathrm{T}_{2}\right)$, and the saturation intensity in excited triplet-triplet channel $\left(\mathrm{T}_{1}-\mathrm{T}_{2}\right)\left(I_{34}^{\text {sat }}\right)$ has a monotonic dependence on radiation intensity $I_{12}$ in principal singlet-singlet channel $\left(\mathrm{S}_{0}-\mathrm{S}_{1}\right)$. Taking into consideration a nonlinear medium with a Gaussian form of coincident mirror-symmetric absorption and emission bands $\left(\omega_{i j}=\omega_{j i} \Rightarrow \delta_{i j}=\left(\omega_{i j}-\omega_{j i}\right) / \Delta_{i j}=0\right)$, where $\Delta_{i j}$ and $\omega_{i j}$ are the profile halfwidth and the centre of $i-j$ chan- nel. For this matter the radiations in principal singletsinglet channel $\left(\mathrm{S}_{0}-\mathrm{S}_{1}\right)$ are tuned at the centre of absorption band $\left(\omega_{0}=\omega_{12}\right)$ and the radiations in excited triplet-triplet channel $\left(\mathrm{T}_{1}-\mathrm{T}_{2}\right)$ are tuned at the centre of absorption band $\left(\omega=\omega_{34}\right)$.

For numerical analysis of Eqs. (2) and (3), the radiation intensities in principal channel $\left(I_{12}\right)$ are normalized to intensity $v\left(P_{21}+P_{23}\right) / B_{12}^{\max }$ and the radiation intensities in excited channel $\left(I_{34}\right)$ are normalized to intensity $v P_{43} / B_{34}^{\max }$. The ratios of spontaneous and radiationless transitions are $P_{43} /\left(P_{21}+P_{23}\right)=P_{23} / P_{31}=10$. At the same time the extinction coefficients in principal and excited channels $\kappa_{12}\left(\omega_{0}\right), \kappa_{34}(\omega)$ are normalized to the value of initial extinction coefficient $\kappa_{0}$ in Figs. 2 and 3. For Fig. 4 the extinction coefficient in excited channel $\kappa_{34}(\omega)$ is normalized to the value $\kappa_{34}^{0}$ of extinction coefficient in excited channel.
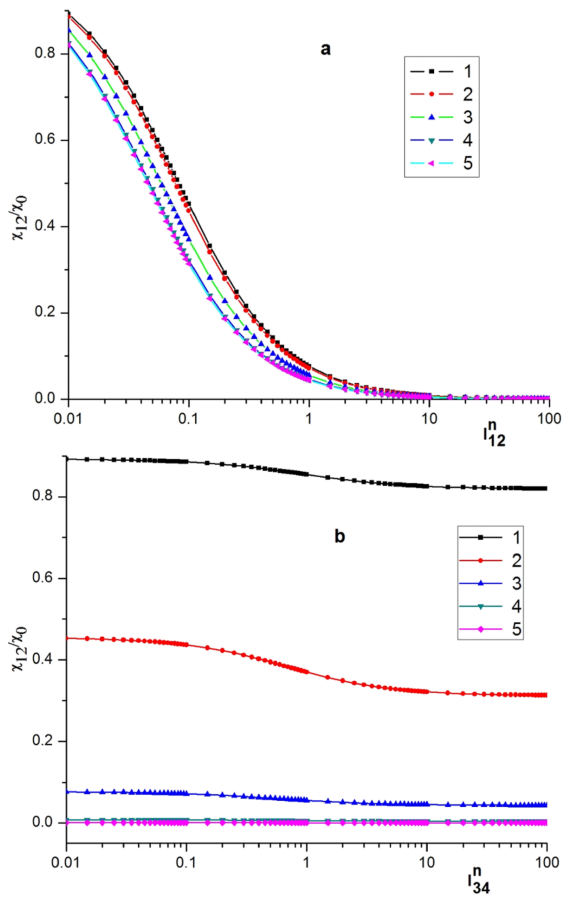

Fig. 2. Dependence of extinction coefficient in principal channel $\chi_{12} / \chi_{0}$ on: radiation intensity: $I_{12}^{n}(\mathrm{a})$, $I_{34}^{n}$ (b). Curves: $1,2,3,4$ and 5 at: (a) $I_{34}^{n}=0.01$, $0.1,1,10$ and 100 , (b) $I_{12}^{n}=0.01,0.1,1,10$ and 100 for frequency tuning $\eta_{12}=\eta_{34}=0$.

Equation (2) and Fig. 2 show the monotonic dependence of extinction coefficient in principal channel $\kappa_{12}\left(\omega_{0}\right)$ on radiation intensity $I_{12}$, Fig. $2 \mathrm{a}$, for different intensities of radiations in excited channel $I_{34}$ and on radiation intensity in the excited channel $I_{34}$, Fig. $2 \mathrm{~b}$, for different intensities of radiations in principal channel $I_{12}$.

Equation (3) and Fig. 3 show the monotonic dependence of extinction coefficient in excited channel $\kappa_{34}(\omega)$ on radiation intensity $I_{34}$, Fig. 3a, for different intensities of radiations in principal channel $I_{12}$. This figure illustrates the monotonic dependence of $\kappa_{34}(\omega)$ on ra- 

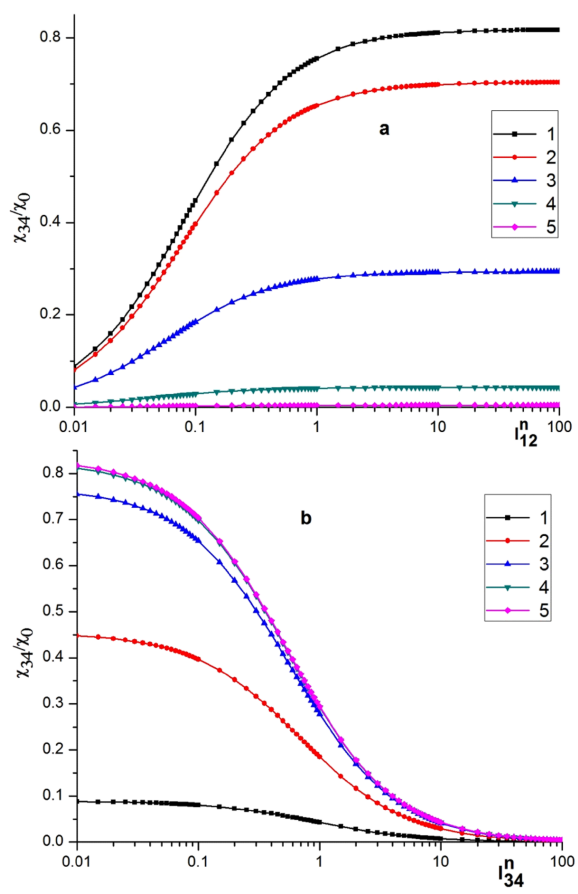

Fig. 3. Dependence of extinction coefficient in excited channel $\chi_{34} / \chi_{0}$ on radiation intensity: $I_{34}^{n}(\mathrm{a}), I_{12}^{n}(\mathrm{~b})$. Curves: $1,2,3,4$ and 5 at: (a) $I_{12}^{n}=0.01,0.1,1,10$ and 100, (b) $I_{34}^{n}=0.01,0.1,1,10$ and 100 for frequency tuning $\eta_{12}=\eta_{34}=0$.

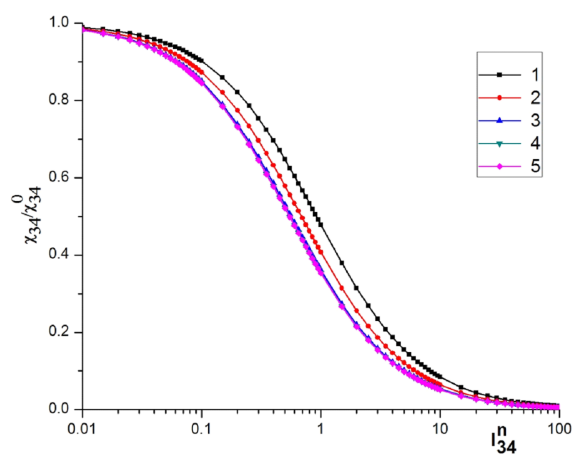

Fig. 4. Dependence of extinction coefficient in excited channel $\chi_{34} / \chi_{34}^{0}$ on radiation intensity: $I_{34}^{n}$. Curves: 1 , 2, 3, 4 and 5 at: $I_{12}^{n}=0.01,0.1,1,10$ and 100 for frequency tuning $\eta_{12}=\eta_{34}=0$.

diation intensity in the principal channel $I_{12}$ for different intensities of radiations in excited channel $I_{34}$. The extinction coefficient in excited channel $\left(\kappa_{34}(\omega)\right)$, which is normalized to initial extinction coefficient in excited channel $\left(\kappa_{34}^{0}\right)$, is illustrated in Fig. 4 . This figure shows the monotonic dependence of extinction coefficient in excited channel $\left(\kappa_{34}(\omega)\right)$ on radiation intensity in this channel $I_{34}$.

\section{Conclusion}

For medium with coincident mirror-symmetric absorption and emission bands, the extinction coefficient in the principal channel decreases with increasing the intensities in both principal and excited channels. At the same time the extinction coefficient in excited channel decreases with increasing the intensity in excited channel but increases with increasing the intensity in principal channel.

The saturation processes, in the given channel, depend on the easy controlled intensity of radiations in the other channel.

\section{Acknowledgments}

This work has been performed during the sabbatical leave of the author (the academic year 2008-2009) in Al-Hussein Bin-Talal University. I would like to thank Tafila Technical University for their support.

\section{References}

[1] A.K. Popov, A.S. Bayev, T.F. George, V.M. Shalaev, Exp. Ph. J. Direct D 1, 1 (2000).

[2] A.S. Rubanov, A.L. Tolstik, S.M. Karpuk, O. Ormachea, Opt. Commun. 181, 183 (2000).

[3] E.A. Tichonov, M.T. Shpak, Nonlinear Optical Effects in Organic Compounds, Naukowa Dumka, Kiev 1979, p. 90, (in Russian).

[4] I.N. Agishev, N.A. Ivanova, A.L. Tolstik, Opt. Commun. 156, 199 (1998).

[5] J.S. Addasi, in: Proc. Second Int. Conf. Lasers in Science and Technology, Jordan, University of Jordan, 1994, p. 39.

[6] J.S. Addasi, J. Appl. Sci. 7, 2896 (2007).

[7] V.V. Kabanov, A.S. Rubinov, A.L. Tolstick, ibid 15, 1681 (1988).

[8] A.L. Tolstic, Ph.D. Thesis, Minsk 1987, p. 138.

[9] E.V. Evakin, S.M. Karpuck, A.S. Rubanov, A.L. Tolstick, A.V. Chaley, Trans. Russian Acad. A Sci. 56, 41 (1992).

[10] M.A. Vasilieva, V.B. Gulbinas, V.I. Kabelka, A.V. Masalov, V.P. Syrus, Sov. J. Quant. Electron. 11, 1431 (1984).

[11] M.A. Vasilieva, YU.K. Vishchakas, V.B. Gulbinas, A. Daugwilla, V.I. Kabelka, ibid 14, 1691 (1987).

[12] J.S. Addasi, in: Proc. First Int. Conf. Institute of Physics, London, 2002, IOP Publishing Ltd, 2003, p. 159.

[13] V.M. Shalaev, Topics in Applied Physics, Springer Verlag, Berlin 2002, p. 82. 